\title{
Research on the Application of BIM in Sewage Treatment Project
}

\author{
Jiayuan Wang, Xiaohan Han, Miao Qun*, Fengjiao Luan
}

School of Environment and Municipal Engineering, Qingdao University of Technology, Qingdao, Shandong, 266033, China

lgdmq@163.com

\begin{abstract}
Keywords: Building information modeling(BIM), Sewage treatment project, Visualization, Construction simulation.

Abstract.As urban water pollution becomes increasingly serious, the sewage treatment process becomes much more complicated, highly complex sewage treatment projects challenge the traditional 2D design and construction management mode. Based on parameterization, visualization, simulation and collaboration, the 4 characteristics of BIM technology, this paper used core softwares of BIM, building sewage treatment structure models, to explore a suitable way for modeling, and based on the 3D building information model, imported the schedule information to form a 4D model, carrying on 4D construction simulation for the model, studied the application of BIM technology in the construction management of sewage treatment project.
\end{abstract}

\section{Introduction}

BIM (Building Information Modeling) is a revolutionary technology which transforms 2D drawing design into 3D digital design. On the basis of 3D digital technology, multifarious engineering data information can be shown by means of visual expression through the use of BIM. BIM is a combination of geometric features, functional requirements and component performance information, and it also includes the project's construction schedule and control information of the construction process.

With the accelerating process of urbanization and industrialization, urban environmental problems, especially the water pollution becomes increasingly serious. Its severity is reflected in the increase of total sewage treatment pressure, the complexity of the sewage composition and the high demand of sewage treatment technology. The national and local governments attach great importance to the construction of urban sewage plant, committed to expand the scale of the sewage plant construction, to improve the sewage treatment process and the efficiency, which makes the design and the construction of sewage treatment project much more difficult. The traditional design and construction management mode, which based on AutoCAD and engineering drawings can not meet the need of highly complex sewage treatment project. The introduction of BIM technology, can provide a good way to solve these problems. 


\section{The main characteristics of BIM}

Parameterization is the most characteristic feature of BIM. The model based on parameterization has a more complete information database, which stores all parameters of the components. No matter how to modify the model, the parameter information has a certain space and a logic relationship with each other.

Visualization means what you see is what you get. The visualization of BIM is a kind of variable scene with interactively formed between components. According to the features, designers can find potential problems in the engineering design or construction organization design in advance, thus the designers can effectively improve design efficiency and quality.

Collaboration is an important feature which makes BIM distinct from CAD. BIM is a data exchange platform rather than a simple model. All participants, including the government, designer, construction side and so on, can insert, extract, edit and update information to solve the problems in the model database.

Simulation makes BIM can simulate buildings, and can do simulate things which can not do in reality. Such as energy-saving simulation, emergency evacuation simulation, construction process simulation are frequently applied to project.

\section{The application of BIM in design phase}

Traditional design work is based on CAD platform, 3D design ideas of designer have to be expressed by 2D drawings, the construction side has to understand the $2 \mathrm{D}$ drawings and transform them to 3D building construction, in this process, the 2D and 3D information transforms for 2 times. The building visualization is poor, inaccurate information expression makes it difficult for different professionals to communicate, which makes error inevitable, if not find it in time, it will lead to great problems in the construction process.

The model created by BIM contains a complete database of the specified project related information. This database contains the size, number, location and other information of all components in a construction project. According to the the model expressed by parametric information, we can see the visual angle which can not be expressed by $2 \mathrm{D}$ drawings. Using the parameterization and visualization of BIM, no matter how complex the structure is, it will be easy to get its accurate flat, facade and profile.

For the problem that sewage treatment structures have a large quantity of special-shaped structures, Revit, a BIM software provides the concept of dimension tool, mainly used to create complex objects. Dimension tool is a kind of race, which makes arbitrary point, line and plane optional to edit. As a special race, it is widely used in drawing special-shaped structures, such as a 
three steps in high density sedimentation basin and a secondary pouring concrete sludge funnel of the sewage treatment plant, the material and structure properties can be defined by this tool as well. As is shown in Figure 1.

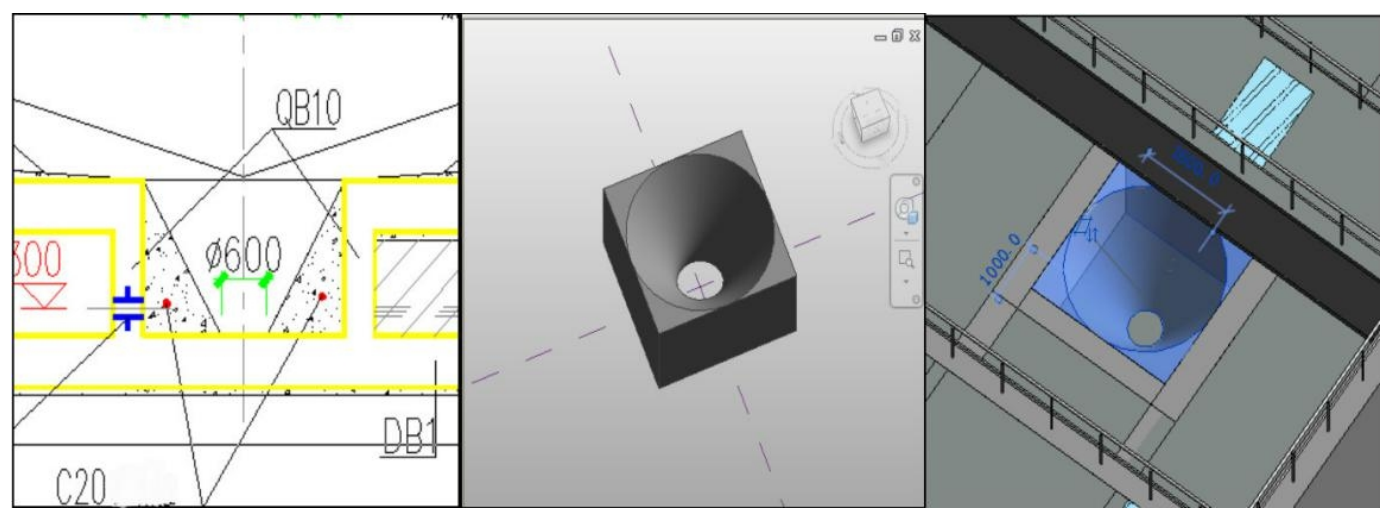

Fig.1 Model of special-shaped structures

The complex structure can be broken down into simple structure parts, respectively to establish parametric models, finally combine them as a whole structure. For example, the high density sedimentation tank is relatively complex, when establish the model, the structure can be broken down into five single parts, dross well, middle corridor, mixing zone, mixing reaction zone and precipitation concentrated tank according to its function. Finally combine these parts together as a whole to get the integrated structure. As is shown in Figure 2.

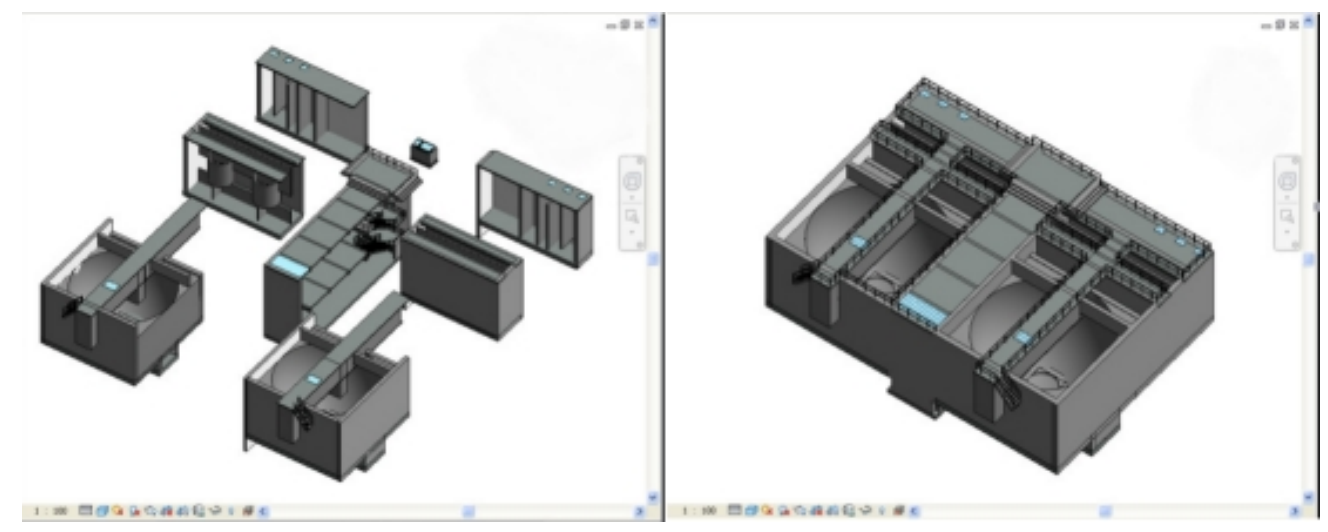

Fig.2 Split figure of the high density sedimentation tank

During the design phase, different professional drawings need to be reviewed, the staff need to analyze the collisions with their experience, which is a waste of manpower, and the efficiency is low. BIM can provide a unified platform to various professionals, to solve the conflict problems. By exporting the Revit model to Naviswoks, and take a collision check on the model, the system will remind designers of the collision problems between individual professional and different professionals, and form a inspection report. Each professional can give way to each other by modifying the only model according to the report. Eliminate the conflicts to provide a model of the optimization for construction side. 


\section{The application of BIM in construction management phase}

Traditional way of construction management is using the form, graphics and other paper documents as a medium, with the aid of different softwares to complete the communication and coordination between all kinds of personnel, such as CTM technology. These methods have many disadvantages, the efficiency is low, the information transfer is not smooth, the charts expression is not so intuitive, which cause many problems in construction phase have to be solved at the last moment. Construction plan is not perfect will easily lead to the construction process in the actual is not consistent with the schedule of materials get into the factory, once the link is wrong, it will eventually lead to confusion in the whole construction plan and cause time lag and waste.

BIM can undertake simulation in multiple stages of construction, by adding the time axis, the 3D model combine with time dimension to carry on a 4D construction simulation. By carrying on the visual simulation of construction progress, problems in the construction can be found in advance, thus improve construction efficiency and reduce the risk of rework, bringing considerable economic benefit for the society.

After completion of the 3D model in the Revit platform, using the data source option in the Navisworks to import the model into Timeliner, and time distribution is arranged in the Animation, then a construction animation flash will be generated, which intuitively reflect the construction process of each working procedure. Create a collection for construction tasks including the structure content, attached the collection to the task, and associated with the construction progress plan, combined with Navisworks virtual animation tool to realize construction process simulation. Based on simulation, one of a characteristics of BIM, the visualization management of the construction process can be implemented. According to the goal of construction progress to constantly update and perfect the scheme, the existing construction scheme will be verified and the operation will be optimized. As is shown in Figure 3.

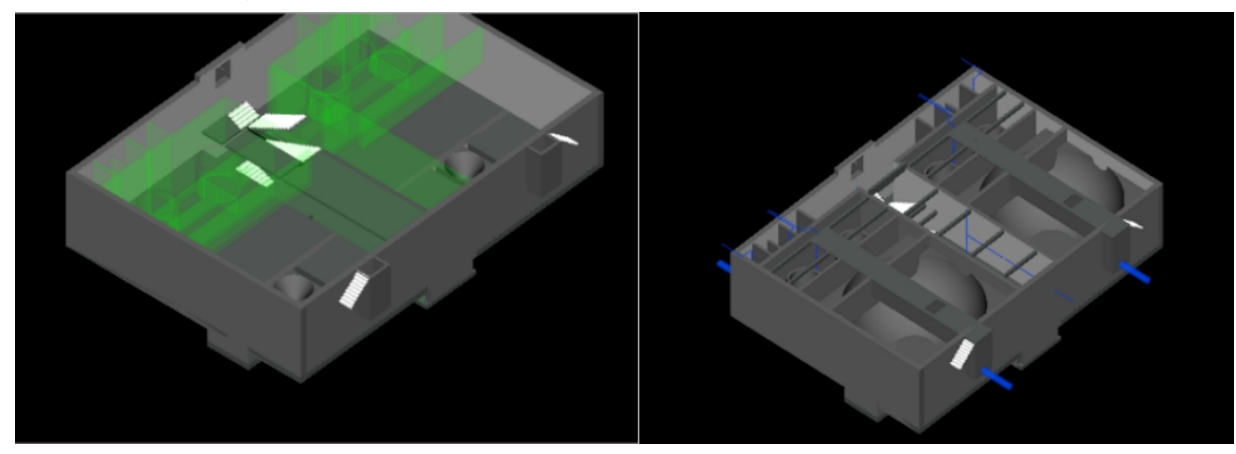

Fig.3 The simulation effect of 3D construction process

\section{Conclusions}

As a product of information technology, BIM keeps up with the trend of the informationization 
development, produces a revolutionary change in construction field. Introducting BIM technology in sewage treatment project can effectively solve the problems of misunderstanding caused by complex special-shaped structures in design phase and the difficult problems of arrangement in construction management phase. BIM fundamentally changes the way to express intent by words and 2D drawings, sets up a good interactive platform for the designers and construction personnel, realizes information sharing and eliminate the wrong information, reduces the cost of resources. Now BIM is mainly applied in large complex building construction projects, the attention in sewage treatment projects is not enough, but the prospect of BIM in the sewage treatment projects shuold not be ignored, and is worth our continuous exploration.

Corresponding author: Miao Qun, Ph.D., professor, municipal engineering.

\section{References}

[1]Gao J, Fischer M . Framework and Case Studies Comparing Implementations and Impacts of 3D/4D Modeling Across Projects[R]. Stanford: Stanford University, 2008

[2]Eastman, C., Teicholz, P.,Sacks, R. and Liston, K. BIM Handbook: A Guide to Building Information Modeling for Owners, Managers, Designers, Engineers and Contractors, New Jersey, John Wiley \& Sons, 2008

[3]Heidari M, Allameh E, DeVries B, etal. Smart-BIM virtual prototype implementation[J]. Automation in Construction, 2014, 39(8): 134-144

[4]Lei Sun. Research and Application of Virtual Technology in Construction[D].Wuhan University of Science and Technology, 2012

[5]Rongbao Wu. Water Treatment Structures Modeling on the Basis of BIM Technology[A]. Engineering Design and Computer Technology: The 15th National Academic Conference of Engineering Design and Computer Application[C] .2010: 363-366 